\title{
Cytoskeleton-associated membrane protein 4 is upregulated in tumor tissues and is associated with clinicopathological characteristics and prognosis in hepatocellular carcinoma
}

\author{
ZHI-YONG CHEN $^{1}$, TING WANG ${ }^{1}$, XIA GAN $^{1,2}$, SI-HAI CHEN $^{1}$, \\ YU-TING HE ${ }^{1}$, YU-QI WANG ${ }^{1}$ and KUN-HE ZHANG ${ }^{1}$ \\ ${ }^{1}$ Department of Gastroenterology, The First Affiliated Hospital of Nanchang University, \\ Jiangxi Institute of Gastroenterology and Hepatology, Nanchang, Jiangxi 330006; \\ ${ }^{2}$ Department of Gastroenterology, The Third Affiliated Hospital of Nanchang University, \\ Nanchang, Jiangxi 330008, P.R. China
}

Received August 11, 2019; Accepted February 21, 2020

DOI: 10.3892/ol.2020.11499

\begin{abstract}
The role of cytoskeleton-associated membrane protein 4 (CKAP4) in hepatocellular carcinoma (HCC) is controversial. The present study aimed to investigate the association between tumor CKAP4 mRNA expression and clinicopathological characteristics and prognosis in patients with HCC. Data relating to CKAP4 mRNA expression in HCC tumor and normal adjacent liver tissues, and clinicopathological characteristics, were downloaded from the Gene Expression Omnibus and The Cancer Genome Atlas databases. The CKAP4 mRNA levels in tumor tissues were compared with those in normal adjacent liver tissues, their association with clinicopathological parameters was analyzed, and diagnostic and prognostic values were evaluated in patients with HCC. In all 4 datasets (total samples, $n=693$ ), CKAP4 mRNA levels were significantly higher in tumor tissues compared with adjacent tissues (all $\mathrm{P}<0.001$ ), with the area under the receiver operating characteristic curve ranging from 0.799-0.898 for HCC diagnosis. In patients with HCC with available clinical data $(n=361)$, the low-level CKAP4 mRNA group exhibited a lower body mass index $(\mathrm{P}=0.005)$, higher $\alpha$-fetoprotein level $(\mathrm{P}<0.001)$, more frequent adjacent liver tissue inflammation $(\mathrm{P}<0.001)$, poorer tumor histological grade $(\mathrm{P}<0.001)$, higher Ishak fibrosis score $(\mathrm{P}=0.035)$ and a more advanced tumor node metastasis $(\mathrm{TNM})$ stage $(\mathrm{P}=0.014)$ compared with the high-level CKAP4 mRNA group. Patients stratified by all the
\end{abstract}

Correspondence to: Professor Kun-He Zhang, Department of Gastroenterology, The First Affiliated Hospital of Nanchang University, Jiangxi Institute of Gastroenterology and Hepatology, 17 Yongwai Zheng Street, Nanchang, Jiangxi 330006, P.R. China E-mail: khzhang@ncu.edu.cn

Key words: hepatocellular carcinoma, cytoskeleton-associated membrane protein 4 mRNA, survival analysis, The Cancer Genome Atlas, biomarker above parameters, except for TNM stage, exhibited significantly different expression of tissue CKAP4 mRNA ( $\mathrm{P}<0.05-0.001)$. Furthermore, higher CKAP4 mRNA levels were observed in patients who died within one year following diagnosis compared with those who survived $>3$ years $(\mathrm{P}=0.003)$. The high-level CKAP4 mRNA group also exhibited lower overall survival (OS) and disease-free survival (DFS) rates compared with the low-level group [hazard ratio $(\mathrm{HR})=1.494 ; 95 \%$ confidence interval (CI), 1.044-2.138; $\mathrm{P}=0.028$ ] for $\mathrm{OS}$ and $(\mathrm{HR}=1.616$; $95 \% \mathrm{CI}, 1.022-2.555 ; \mathrm{P}=0.040)$ for DFS. The results of the present study suggest that CKAP4 mRNA is upregulated in $\mathrm{HCC}$ tumor tissues compared with normal adjacent tissues, and is associated with poor clinical prognosis, pathological features and survival in patients with HCC. Thus, CKAP4 is a potential biomarker for HCC diagnosis and prognosis.

\section{Introduction}

Hepatocellular carcinoma (HCC), representing $75-85 \%$ of primary hepatic carcinoma, was the fourth most frequent cause of cancer-associated mortality worldwide in 2018 (1). There were $\sim 854,000$ new liver cancer cases and $\sim 810,000$ deaths globally in 2015 (2), with nearly equal rates of incidence and mortality. Although, great advances have been made in surgical and non-surgical treatments in the last decades, the outcome of HCC remains poor (3) and $>70 \%$ of patients experience recurrence within 5 years $(4,5)$. The poor prognosis of $\mathrm{HCC}$ is primarily ascribed to delayed diagnosis (6).

The causes of HCC occurrence are complex and involve the accumulation of genetic and epigenetic changes (6). Cellular events are often accompanied by upregulated expression of multiple factors (such as oncogenes) that influence the survival of tumor cells by inhibiting apoptosis and regulating the cell cycle (6).

Cytoskeleton-associated membrane protein 4 (CKAP4; also referred to as P63), is a 63-kDa type II transmembrane protein located in the endoplasmic reticulum $(7,8)$. CKAP-4 plays a vital role in maintaining the structure of the endoplasmic reticulum (8-10). Other studies have identified CKAP4 as a 
cell surface receptor for surfactant protein A, antiproliferative factor and Dickkopf 1 (DKK1) (11-14), which are associated with tumor growth in cervical and bladder cancer $(12,13)$. As a novel DKK1 receptor that stimulates tumor cell proliferation, CKAP4 is frequently upregulated in pancreatic cancer, lung cancer and esophageal squamous cell carcinoma (ESCC), and blocking CKAP4 activity via knockdown or anti-CKAP4 antibodies can prevent xenograft tumor formation (14-16). CKAP4 has also been demonstrated to be upregulated in chronic lymphocytic leukemia (CLL) (17). Recently, exosomal CKAP4 was reported to be a promising biomarker for pancreatic ductal adenocarcinoma (PDAC) (18).

In 2013, Li et al (19) first revealed that CKAP4 was associated with progression and metastasis in patients with HCC. CKAP4 expression was demonstrated to be significantly higher in HCC tissues compared with adjacent normal tissues, and was associated with tumor size, intrahepatic metastasis, portal venous invasion and tumor stage; furthermore, upregulated CKAP4 expression increased overall survival (OS) and disease-free survival (DFS) times in patients with HCC. Other studies have revealed that high CKAP4 expression in HCC cells is associated with low proliferative capacity and invasion potential via the suppression of epithelial growth factor receptor (EGFR) signaling (20), which is involved in HCC occurrence and development (6). The serum CKAP4 concentration in patients with HCC was significantly higher compared with patients with chronic hepatitis B and cirrhosis, as well as healthy controls, which suggests that CKAP4 is a promising indicator for HCC diagnosis, especially when combined with $\alpha$-fetoprotein (AFP) (21). In addition, upregulated CKAP4 expression was observed in HCC in a proteomic study using isobaric tags for relative and absolute quantification (22).

The above studies present contradictory results; upregulated CKAP4 expression in HCC is reported to be associated with low growth and favorable prognosis, but in other cancers, CKAP4 expression appears to promote tumor growth and result in worse prognosis. Additionally, an immunohistochemical study comparing CKAP4 protein expression in cholangiocarcinoma and HCC tissues demonstrated no CKAP4 protein expression in HCC (23).

In the present study, the association between CKAP4 mRNA expression and HCC was investigated using four CKAP4 datasets of cohorts of patients with HCC from the Gene Expression Omnibus (GEO) and The Cancer Genome Atlas (TCGA) databases. The data obtained were used to determine the CKAP4 mRNA expression levels in HCC tumor tissues and adjacent liver tissues, and their diagnostic potential. Associations between CKAP4 mRNA expression and clinicopathological characteristics and the prognosis of patients with HCC were then analyzed.

\section{Materials and methods}

Tissue sample data sources. Three gene expression profile datasets of HCC tumor and adjacent tissues, GSE76427 (24), GSE84402 (25) and GSE57957 (26), were downloaded from the GEO database (https://www.ncbi.nlm.nih.gov/) (Fig. 1) and analyzed using software packages in RStudio 3.6.1 version (https://rstudio.com/). The packages 'illuminaHumanv4 db' (https://bioconductor.org/packages/illuminaHumanv4 db/) and 'hgu133plus2 db' (https://bioconductor.org/packages/ hgu133plus $2 \mathrm{db} /$ ) were used for gene annotation. The aforementioned packages were used to convert gene IDs into gene symbols for subsequent analysis. The packages 'dplyr' (http:// dplyr.tidyverse.org/) and 'tibble' (http://tibble.tidyverse.org/) were used to preprocess the data and the package 'limma' (https://bioconductor.org/packages/limma/) was used to determine whether the data required normalization. $\log _{2}$ normalization was performed for the extracted data. Detailed information regarding tissue samples in the three datasets is presented in Fig. 1.

In addition, a liver cancer (LIHC) dataset with RNAseq data was downloaded from TCGA database (https://portal.gdc. cancer.gov/) and consisted of HCC tumor $(n=374)$ and adjacent tissue samples $(n=50)$. LIHC phenotype data were obtained from the GDC TCGA-LIHC cohort (https://gdc.xenahubs.net/), which contained 469 cases. All the data were processed using software packages in the R program, including 'dplyr', 'tidyr' (http://tidyr.tidyverse.org/), 'DESeq2' (https://bioconductor. org/packages/DESeq2/) and 'edgeR' (https://bioconductor.org/ packages/edgeR/). $\log _{2}(\operatorname{count}+1)$ normalization of the CKAP4 mRNA expression data was achieved with 'DESeq2' and 'vst'. Finally, $361 \mathrm{HCC}$ cases with matched CKAP4 mRNA and clinical data were evaluated (Fig. 1).

Presentation of CKAP4 mRNA levels and the diagnostic value of CKAP4 mRNA in patients with HCC. Differences in CKAP4 mRNA levels between tumor and adjacent normal tissues in the four datasets are presented as expression heatmaps and violin plots. The software package 'pheatmap' (https:// CRAN.R-project.org/package=pheatmap) in the $\mathrm{R}$ program was used to plot the heatmaps and the packages 'ggstatsplot' (https://indrajeetpatil.github.io/ggstatsplot/) and 'ggpubr' (https://rpkgs.datanovia.com/ggpubr/) were used to plot the violin plots. The diagnostic value of the CKAP4 mRNA levels in $\mathrm{HCC}$ was evaluated using a receiver operating characteristic (ROC) curve.

Analysis of the association between CKAP4 mRNA levels and clinicopathological characteristics of patients with HCC. Using patient samples with complete clinical data from TCGA-LIHC dataset, the association between CKAP4 mRNA levels in tumor tissues and clinical characteristics was analyzed. The patients were divided into high- and low-level CKAP4 mRNA groups using the median CKAP4 mRNA level as the cut-off value, and differences in clinical parameters were compared between the 2 groups. In addition, the 'ggboxplot' function in the 'ggpubr' package of the $\mathrm{R}$ program was used to present and compare differences in CKAP4 mRNA expression based on clinical parameters, demonstrating significant differences between the initial high- and low-level CKAP4 mRNA groups.

Survival analyses associated with CKAP4 mRNA levels in patients with HCC. Survival analyses were performed in the cohort of 361 patients with HCC from TCGA-LIHC dataset. In this analysis, cases with a follow-up time of $<30$ days after curative surgical resection were excluded. Univariate Cox regression analysis, was used to evaluate the hazard ratios (HRs), confidence intervals (CI) and P-values of CKAP4 mRNA and the clinicopathological parameters for 


\section{Three HCC datasets from GEO database}
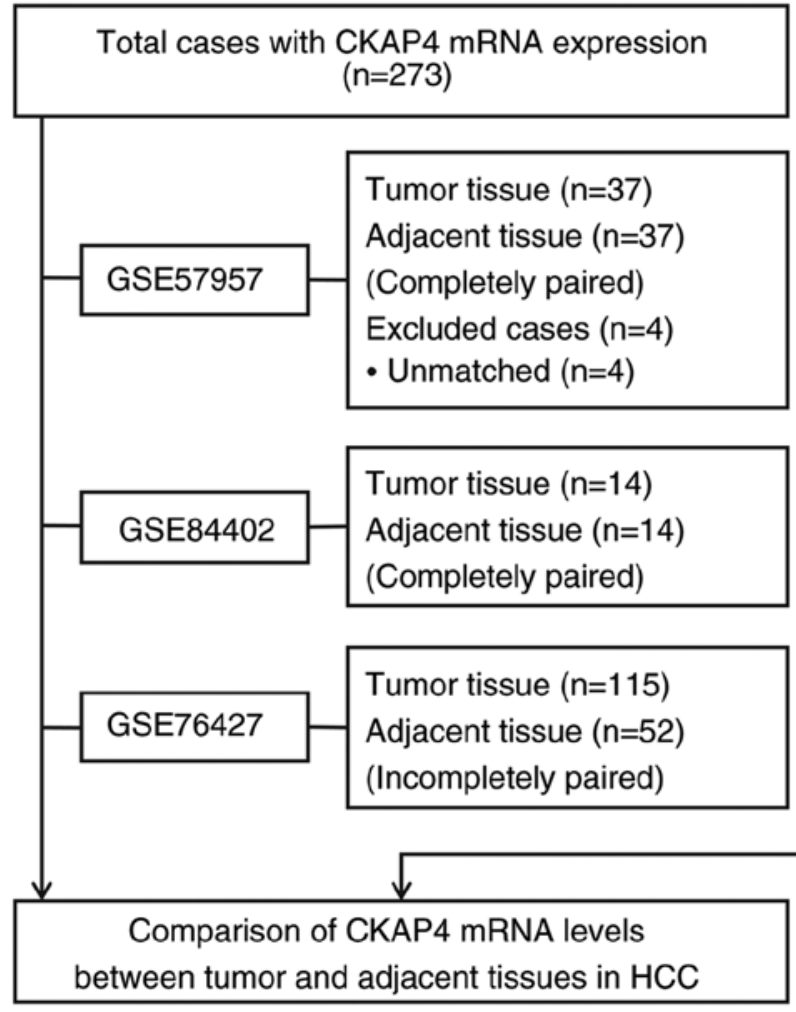

A HCC dataset from TCGA LIHC database

Total cases with gene expression RNAseq $(n=424)$

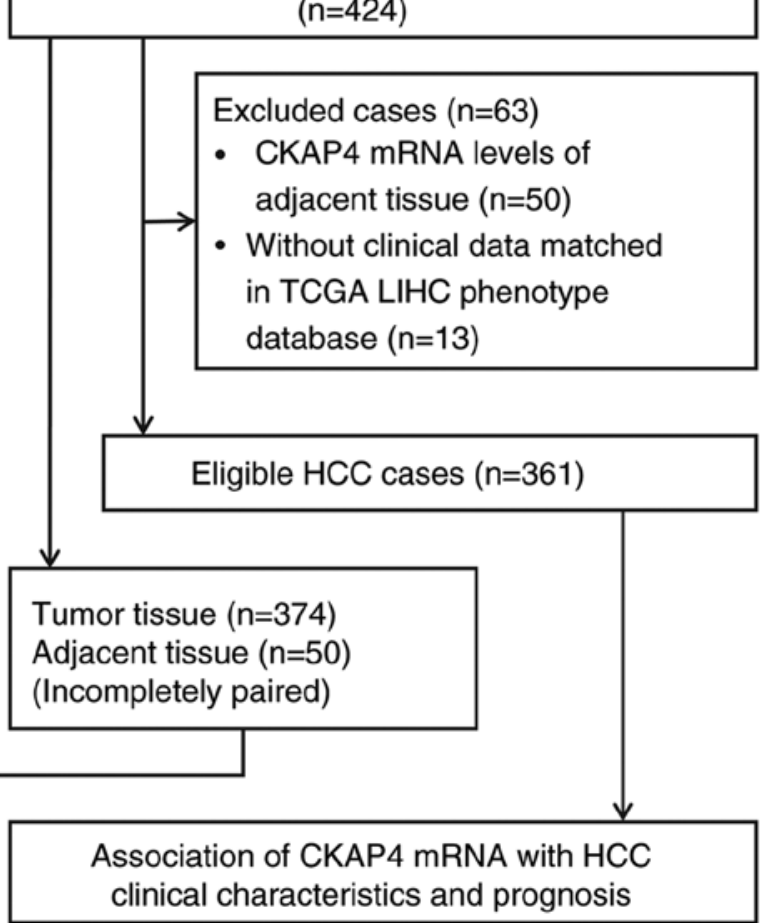

Figure 1. Flow chart providing details of the datasets, including tissue or patient numbers, used in the present study. HCC, hepatocellular carcinoma; GEO, Gene Expression Omnibus; TCGA-LIHC, The Cancer Genome Atlas-Liver Cancer database; CKAP4, cytoskeleton-associated membrane protein 4.

OS and DFS. However, multivariate Cox regression analysis was not conducted owing to an insufficient number of cases with complete data. Kaplan-Meier survival analysis of the predictive power of CKAP4 mRNA levels for OS and DFS of patients with $\mathrm{HCC}$ after hepatectomy was performed using the packages 'survival' (https://github.com/therneau/survival/) and 'survminer' (https://rpkgs.datanovia.com/survminer/) in R. CKAP4 mRNA expression levels were compared among patients with different survival times and among patients with different recurrence times after hepatectomy using the function 'ggboxplot' in the 'ggpubr' packages in R software.

Statistical analysis. Data are presented as the mean $\pm \mathrm{SD}$, median and interquartile range (IQR), or constituent ratio, according to the data type. The paired t-test (for groups with completely paired tumor and adjacent samples), unpaired t-test (for independent groups) and $\chi^{2}$ test were used to compare CKAP4 mRNA levels between two groups and calculated using R. One-way ANOVA followed by Bonferroni's post-hoc test was used for comparing differences in the CKAP4 mRNA levels between multiple groups, and calculated using SPSS Statistics software v.24.0 (IBM Corp.). Ridit analysis and the Mann-Whitney $U$ test were used to compare differences in clinicopathological parameters between the low- and high-level CKAP4 mRNA groups, and calculated using SPSS v.24.0. Kaplan-Meier curves were generated and compared using the Tarone-Ware test (for survival plots where crossover between the groups is observed) or log-rank test in R. Univariate Cox regression analysis was used to calculate HRs and their $95 \%$ CIs, as well as the associated $\mathrm{P}$ values. A two-sided $\mathrm{P}<0.05$ was considered to indicate a statistically significant difference.

\section{Results}

CKAP4 mRNA levels are elevated in tumor tissues compared with adjacent liver tissues in patients with HCC. Heat maps and violin plots were used to intuitively display CKAP4 mRNA expression in tumor and adjacent liver tissues, and areas under ROC curves were used to evaluate the diagnostic value for HCC. Heat maps of CKAP4 mRNA levels in all four datasets demonstrate elevated ('hot') expression in tumor tissues and reduced ('cold') expression in adjacent liver tissues (Fig. 2A-D). Compared with adjacent liver tissues, tumor tissues from patients with HCC exhibit significantly upregulated CKAP4 mRNA levels (following normalization); furthermore, CKAP4 mRNA demonstrated moderate power for the diagnosis of HCC in all of the datasets, with AUCs ranging from $0.799-0.898$, indicating its potential diagnostic value in $\mathrm{HCC}$ (Fig. 2A-D).

Association between CKAP4 mRNA levels and clinical characteristics of patients with HCC. Clinicopathological and demographic characteristics were obtained for the 

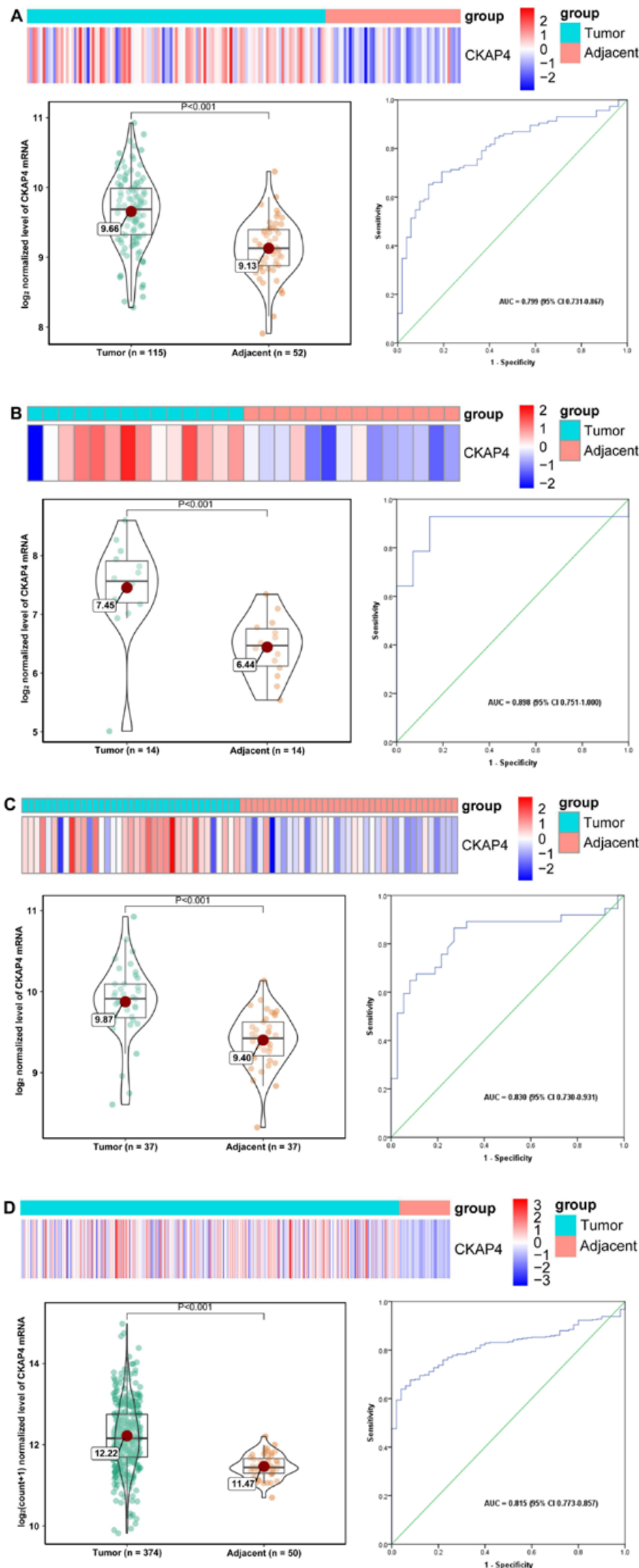

Figure 2. CKAP4 mRNA expression heat maps and levels in tumor tissues and adjacent liver tissues (violin plots) and the corresponding receiver operating curves for diagnosing HCC in the (A) GSE76427, (B) GSE84402, (C) GSE57957 and (D) TCGA-LIHC datasets from the GEO and TCGA databases. In the heat maps, red represents upregulation of CKAP4 mRNA and blue represents downregulation of CKAP4 mRNA; numbers in the center of the violin plots representing the median value of CKAP4 mRNA. HCC, hepatocellular carcinoma; GEO, Gene Expression Omnibus; TCGA-LIH, The Cancer Genome AtlasLiver Cancer database; CKAP4, cytoskeleton-associated membrane protein 4; AUC, area under curve; CI, confidence interval. 
A

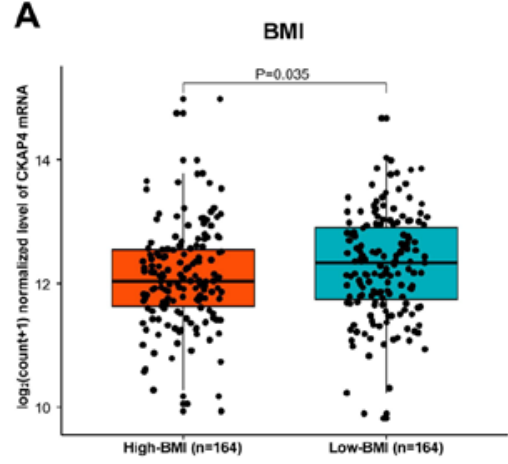

B

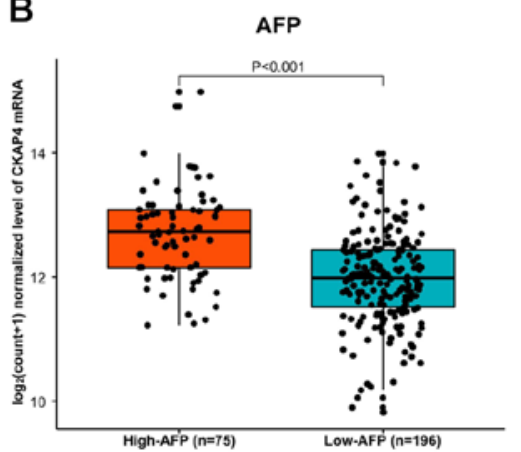

C

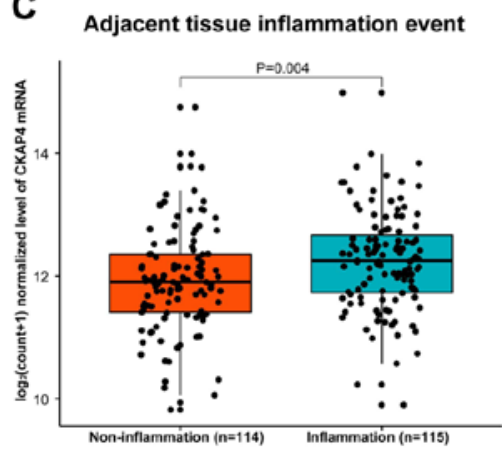

D

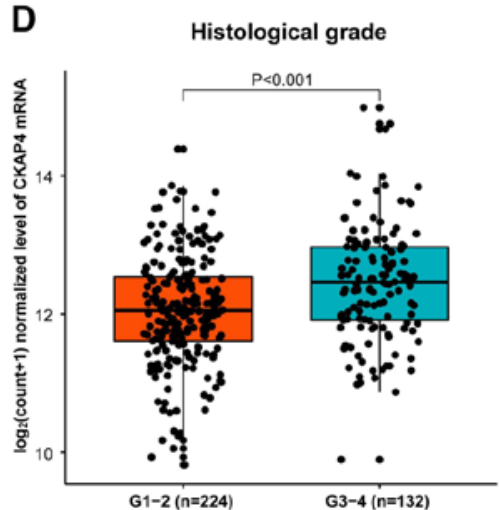

E

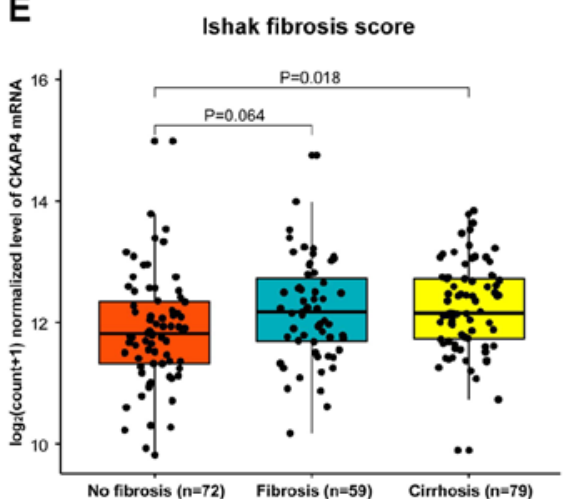

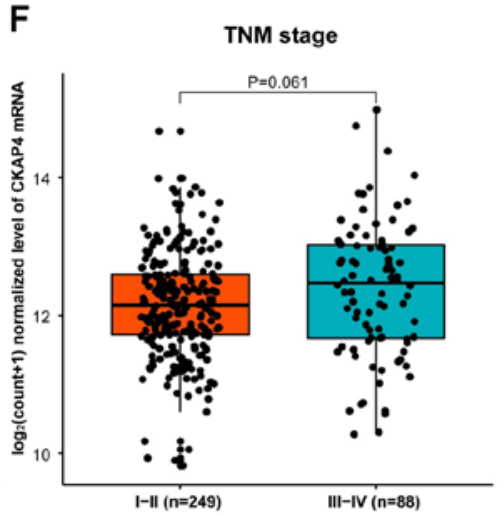

Figure 3. Comparisons of CKAP4 mRNA levels between subgroups of 6 significant clinicopathological parameters. (A) Stratified into high- (>median) and low-BMI ( $\leq$ median) groups using the median BMI value. (B) Stratified by AFP value into high- ( $>200 \mathrm{ng} / \mathrm{ml})$ and low-AFP ( $\leq 200 \mathrm{ng} / \mathrm{ml})$ groups. (C) Stratified by tumor adjacent liver tissue inflammation event into non-inflammation and inflammation groups. (D) Stratified by tumor histological grade into G1-2 and G3-4 groups. (E) Stratified by Ishak fibrosis score into no fibrosis (0), fibrosis (1-4), and cirrhosis (5-6) groups. (F) Stratified by TNM stage into I-II and III-IV groups. BMI, body mass index; AFP, $\alpha$-fetoprotein; TNM, tumor-node-metastasis; CKAP4, cytoskeleton-associated membrane protein 4.

361 patients with HCC from TCGA database, and are summarized in Table I. Body mass index $(\mathrm{BMI})(\mathrm{P}=0.005)$, AFP level $(\mathrm{P}<0.001)$, tumor histological grade $(\mathrm{P}<0.001)$, adjacent liver tissue inflammation event $(\mathrm{P}<0.001)$, Ishak fibrosis score $(\mathrm{P}=0.035)$, and American Joint Committee on Cancer (AJCC) tumor node metastasis (TNM) stage $(\mathrm{P}=0.014)$ were associated with CKAP4 mRNA levels, as they significantly differed between the high- and low-level CKAP4 mRNA groups (Table I). The CKAP4 mRNA levels were further compared between subgroups for each of these parameters (Fig. 3), and the results demonstrated that CKAP4 mRNA levels in tumor tissues were significantly different between the subgroups for all five parameters, except for AJCC TNM stage (Fig. 3).

High CKAP4 mRNA levels are associated with poor prognosis in patients with HCC. Using univariate Cox regression analysis, associations between CKAP4 mRNA levels in tumor tissues and the clinicopathological parameters considered as risk factors for the survival of HCC patients were evaluated, and the results are summarized in Table II. High CKAP4 mRNA levels, macrovascular invasion and TNM III-IV stage are risk factors for both OS and DFS in patients with HCC (Table II). BMI was a protective factor for DFS and hepatitis $\mathrm{B}$ was a protective factor for OS and DFS with reference to alcohol consumption (Table II).
HCC patient survival was compared between the high- and low-level CKAP4 mRNA groups and the results demonstrated significantly lower OS and DFS probabilities in the high-level group (Fig. 4A and B). Additionally, CKAP4 mRNA levels were significantly different between the patients with survival times of $<1$ year and $>3$ years $(\mathrm{P}=0.003$; Fig. $4 \mathrm{C})$, and between the patients with a recurrence times $<1$ year and $\geq 1$ year $(\mathrm{P}=0.046$; Fig. 4D), indicating that higher CKAP4 mRNA levels are associated with poor prognosis.

\section{Discussion}

In the present study, CKAP4 mRNA levels in tumor and adjacent liver tissues from patients with HCC were compared, their associations with clinicopathological parameters were analyzed, and their potential diagnostic and prognostic values for HCC were evaluated. The present study demonstrated that CKAP4 mRNA levels were significantly upregulated in tumor tissues [with a moderate area under curve (AUC) for HCC diagnosis], and that this was significantly associated with poor clinicopathological parameters (lower BMI, higher AFP, adjacent liver tissue inflammation event, poor tumor histological grade, higher Ishak fibrosis score, and advanced AJCC TNM stage) and with poor prognosis in patients with HCC (lower OS and DFS survival probabilities, shorter survival and recurrence times after tumor resection). 
Table I. Differences in clinicopathological and demographic characteristics between high- and low-level CKAP4 mRNA groups in patients with HCC.

\begin{tabular}{|c|c|c|c|c|}
\hline \multirow[b]{2}{*}{ Characteristic } & \multirow[b]{2}{*}{ Patients, $\mathrm{n}$} & \multicolumn{2}{|c|}{ CKAP4 mRNA group } & \multirow[b]{2}{*}{ P-value } \\
\hline & & Low-level & High-level & \\
\hline Age, (years) n, median (IQR) & 360 & $\begin{array}{c}181,62.0 \\
(53.0-69.0)\end{array}$ & $\begin{array}{c}179,60.0 \\
(51.0-68.0)\end{array}$ & 0.119 \\
\hline Sex, n $(\%)$ & 361 & & & 0.085 \\
\hline Male & 244 & $130(53.3)$ & $114(46.7)$ & \\
\hline Female & 117 & $51(43.6)$ & $66(56.4)$ & \\
\hline Ethnicity, n (\%) & 349 & & & 0.071 \\
\hline Asian & 156 & $68(43.6)$ & $88(56.4)$ & \\
\hline White & 176 & $97(55.1)$ & $79(44.9)$ & \\
\hline Black or African American & 17 & $8(47.1)$ & $9(52.9)$ & \\
\hline History of risk factors, $\mathrm{n}(\%)$ & 342 & & & 0.803 \\
\hline Alcohol consumption & 66 & $36(54.5)$ & $30(45.5)$ & \\
\hline Hepatitis B & 94 & $41(43.6)$ & $53(56.4)$ & \\
\hline Hepatitis C & 51 & $27(52.9)$ & $24(47.1)$ & \\
\hline Non-alcoholic fatty liver disease & 17 & $9(52.9)$ & $8(47.1)$ & \\
\hline Other & 28 & $12(42.9)$ & $16(57.1)$ & \\
\hline None & 86 & $46(53.5)$ & $40(46.5)$ & \\
\hline Family history of cancer, $\mathrm{n}(\%)$ & 312 & & & 0.196 \\
\hline No & 203 & $98(48.3)$ & $105(51.7)$ & \\
\hline Yes & 109 & $61(56.0)$ & $48(44.0)$ & \\
\hline Body mass index, $\mathrm{kg} / \mathrm{m}^{2}, \mathrm{n}$, median (IQR) & 328 & $\begin{array}{c}167,25.3 \\
(22.4-29.85)\end{array}$ & $\begin{array}{c}161,23.6 \\
(21.2-27.5)\end{array}$ & 0.005 \\
\hline$\alpha$-fetoprotein, $\mathrm{ng} / \mathrm{ml}, \mathrm{n}$, median (IQR) & 271 & $7(3-47)$ & $35(8-2466)$ & $<0.001$ \\
\hline$\alpha$-fetoprotein, $\mathrm{ng} / \mathrm{ml}, \mathrm{n}(\%)$ & 271 & & & $<0.001$ \\
\hline$<200$ & 196 & $121(61.7)$ & $75(38.3)$ & \\
\hline$\geq 200$ & 75 & $21(28.0)$ & $54(72.0)$ & \\
\hline Adjacent liver tissue inflammation event, $\mathrm{n}(\%)$ & 229 & & & $<0.001$ \\
\hline No & 114 & $78(68.4)$ & $36(31.6)$ & \\
\hline Yes & 115 & $52(45.2)$ & $63(54.8)$ & \\
\hline Tumor histological grade, $\mathrm{n}(\%)$ & 356 & & & $<0.001$ \\
\hline G1 & 53 & $35(66.0)$ & $18(34.0)$ & \\
\hline $\mathrm{G} 2$ & 171 & $93(54.4)$ & $78(45.6)$ & \\
\hline G3 & 121 & $47(38.8)$ & $74(61.2)$ & \\
\hline G4 & 11 & $3(27.3)$ & $8(72.7)$ & \\
\hline Vascular invasion, $\mathrm{n}(\%)$ & 305 & & & 0.215 \\
\hline None & 200 & $108(54.0)$ & $92(46.0)$ & \\
\hline Micro & 89 & $42(47.2)$ & $47(52.8)$ & \\
\hline Macro & 16 & $7(43.8)$ & $9(56.2)$ & \\
\hline Ishak fibrosis score, $\mathrm{n}(\%)$ & 210 & & & 0.035 \\
\hline 0, no fibrosis & 72 & $50(69.4)$ & $22(30.6)$ & \\
\hline 1-4, fibrosis & 59 & $29(49.2)$ & $30(50.8)$ & \\
\hline 5-6, cirrhosis & 79 & $41(51.9)$ & $38(48.1)$ & \\
\hline AJCC TNM stage, n (\%) & 337 & & & 0.014 \\
\hline I & 167 & $92(55.1)$ & $75(44.9)$ & \\
\hline II & 82 & $38(46.3)$ & $44(53.7)$ & \\
\hline III & 84 & $35(41.7)$ & $49(58.3)$ & \\
\hline IV & 4 & $0 \quad(0.0)$ & $4(100.0)$ & \\
\hline
\end{tabular}


Table I. Continued.

\begin{tabular}{lcccr}
\hline & & \multicolumn{2}{c}{ CKAP4 mRNA group } & \\
Characteristic & Patients, $\mathrm{n}$ & Low-level & High-level & P-value \\
\hline Recurrence type, $\mathrm{n}(\%)$ & 152 & & & 0.400 \\
Extrahepatic & 39 & $21(53.8)$ & $18(46.2)$ & \\
Intrahepatic & 64 & $32(50.0)$ & $32(50.0)$ & \\
Locoregional & 49 & $22(44.9)$ & $27(55.1)$ & \\
\hline
\end{tabular}

CKAP4 mRNA low- and high-level groups were stratified by the median value of CKAP4 mRNA expression. CKAP4, cytoskeleton-associated membrane protein 4; HCC, hepatocellular carcinoma; IQR, inter-quartile range; AJCC, American Joint Committee on Cancer; TNM, tumornode-metastasis.
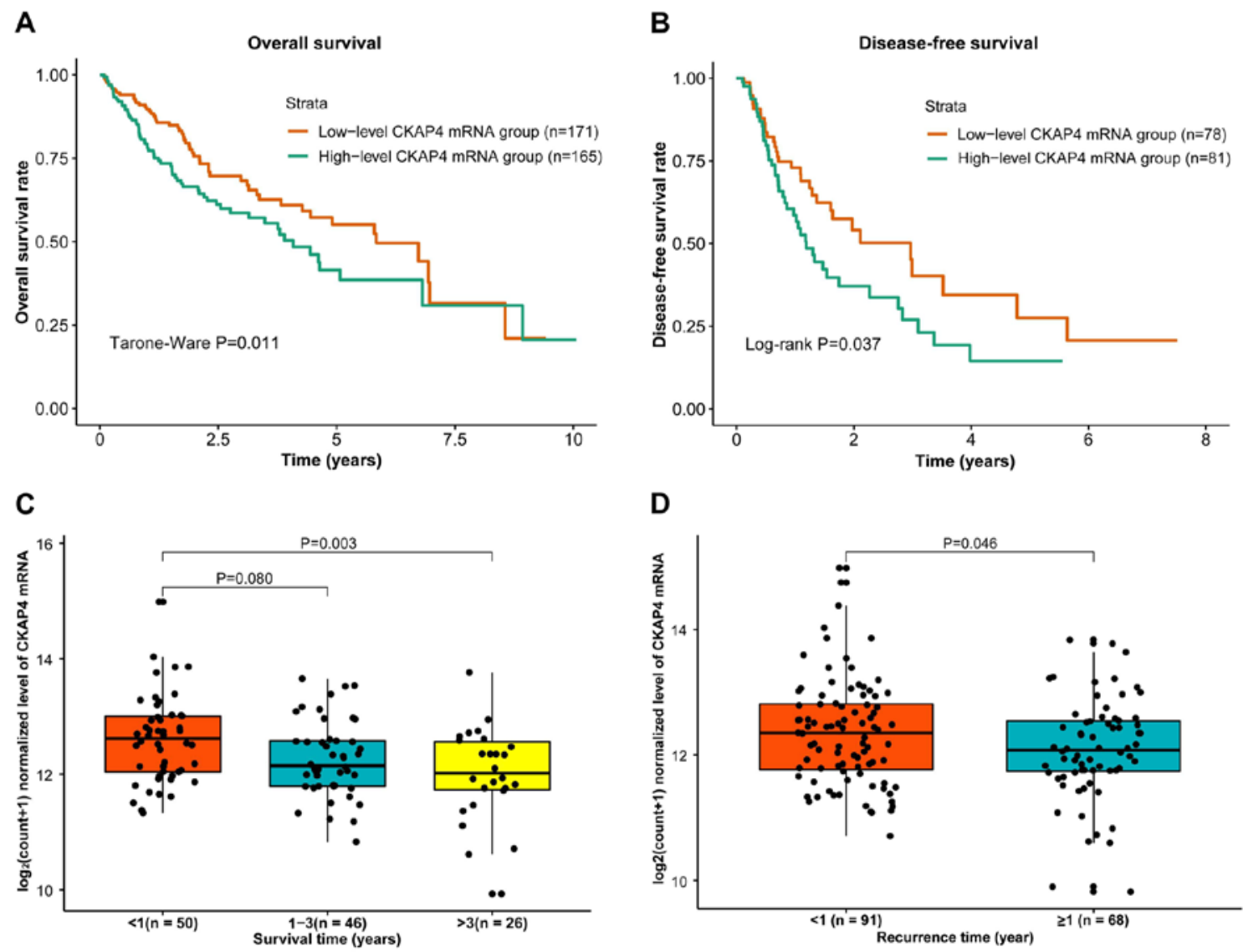

Figure 4. Association between CKAP4 mRNA level and survival in patients with HCC. Kaplan-Meier curves for the (A) overall survival (B) disease-free survival of patients with HCC, stratified according to the median CKAP4 mRNA level. (C) Scatter box plots of CKAP4 mRNA levels against survival times of $<1$ year, 1-3 years and $>3$ years in patients with HCC. (D) Scatter box plots of CKAP4 mRNA levels against recurrence times of $<1$ year and $\geq 1$ year in patients with HCC. CKAP4, Cytoskeleton-associated membrane protein 4; HCC, hepatocellular carcinoma.

Data were extracted from four independent HCC datasets from the GEO and TCGA databases, and the CKAP4 mRNA levels in tumor and adjacent liver tissues from patients with HCC were compared. All four datasets showed consistent results, indicating that CKAP4 mRNA levels were significantly higher in tumor tissues compared with adjacent liver tissues. These results confirm the upregulation of CKAP4 in tumor tissues in accordance with previous results found in HCC (19) and other types of cancer, including cervical cancer, bladder cancer, lung cancer, pancreatic cancer,
ESCC, CLL, PDAC and intrahepatic cholangiocellular carcinoma $(12-18,27)$.

CKAP4 upregulation in HCC tissues makes it a potential diagnostic and prognostic biomarker in HCC. In the present study, CKAP4 mRNA provided moderate power for the diagnosis of HCC, with AUCs ranging from 0.799-0.898 for HCC diagnosis. To the best of our knowledge, similar studies involving the diagnostic evaluation of CKAP4 mRNA for HCC have not been conducted; however, Wang et al (21) reported that the serum concentration of CKAP4 protein 
Table II. Associations of CKAP4 mRNA level and other metrics with OS and DFS in patients with HCC.

\begin{tabular}{|c|c|c|c|c|}
\hline \multirow[b]{2}{*}{ Variables } & \multicolumn{2}{|c|}{ Cox regression for OS } & \multicolumn{2}{|c|}{ Cox regression for DFS } \\
\hline & $\mathrm{HR}(95 \% \mathrm{CI})$ & P-value & $\mathrm{HR}(95 \% \mathrm{CI})$ & P-value \\
\hline \multicolumn{5}{|l|}{ CKAP4 mRNA } \\
\hline Low-level & Reference & & Reference & \\
\hline High-level & $1.494(1.044-2.138)$ & 0.028 & $1.616(1.022-2.555)$ & 0.040 \\
\hline Age, years & $1.007(0.993-1.021)$ & 0.307 & $1.005(0.978-1.023)$ & 0.595 \\
\hline \multicolumn{5}{|l|}{ Sex } \\
\hline Male & Reference & & Reference & \\
\hline Female & $0.810(0.562-1.167)$ & 0.257 & $0.744(0.471-1.177)$ & 0.207 \\
\hline \multicolumn{5}{|l|}{ Race } \\
\hline Asian & Reference & & Reference & \\
\hline White & $1.304(0.885-1.919)$ & 0.179 & $0.995(0.615-1.609)$ & 0.984 \\
\hline Black or African American & $1.365(0.540-3.455)$ & 0.511 & NA & \\
\hline \multicolumn{5}{|l|}{ History of risk factors } \\
\hline Alcohol consumption & Reference & & Reference & \\
\hline Hepatitis B & $0.258(0.136-0.487)$ & $<0.001$ & $0.471(0.226-0.985)$ & 0.045 \\
\hline Hepatitis C & $0.854(0.474-1.538)$ & 0.600 & $0.580(0.269-1.251)$ & 0.165 \\
\hline Non-alcoholic fatty liver disease & $0.403(0.123-1.325)$ & 0.135 & $0.787(0.236-2.628)$ & 0.698 \\
\hline Other & $0.615(0.282-1.345)$ & 0.224 & $0.695(0.300-1.610)$ & 0.396 \\
\hline None & $1.069(0.667-1.712)$ & 0.782 & $1.260(0.702-2.262)$ & 0.439 \\
\hline \multicolumn{5}{|l|}{ Family history of cancer } \\
\hline No & Reference & & Reference & \\
\hline Yes & $1.157(0.793-1.693)$ & 0.454 & $1.314(0.822-2.10)$ & 0.255 \\
\hline \multicolumn{5}{|l|}{$\alpha$-fetoprotein, $\mathrm{ng} / \mathrm{ml}$} \\
\hline$<200$ & Reference & & Reference & \\
\hline$\geq 200$ & $1.123(0.689-1.829)$ & 0.642 & $1.818(0.935-3.535)$ & 0.078 \\
\hline \multicolumn{5}{|c|}{ Adjacent liver tissue inflammation event } \\
\hline No & Reference & & Reference & \\
\hline Yes & $1.242(0.752-2.052)$ & 0.397 & $0.927(0.504-1.704)$ & 0.806 \\
\hline \multicolumn{5}{|l|}{ Tumor histological grade } \\
\hline G1 & Reference & & Reference & \\
\hline G2 & $1.220(0.710-2.096)$ & 0.471 & $1.325(0.664-2.645)$ & 0.425 \\
\hline G3-G4 & $1.195(0.681-2.098)$ & 0.535 & $1.789(0.891-3.592)$ & 0.102 \\
\hline \multicolumn{5}{|l|}{ Vascular invasion } \\
\hline None & Reference & & Reference & \\
\hline Micro & $1.366(0.858-2.175)$ & 0.189 & $1.159(0.610-2.203)$ & 0.652 \\
\hline Macro & $2.575(1.215-5.457)$ & 0.014 & $3.104(1.178-8.181)$ & 0.022 \\
\hline \multicolumn{5}{|l|}{ Ishak fibrosis score } \\
\hline 0, No fibrosis & Reference & & Reference & \\
\hline 1-4, Fibrosis & $0.749(0.381-1.472)$ & 0.403 & $0.589(0.267-1.300)$ & 0.190 \\
\hline 5-6, Cirrhosis & $0.732(0.396-1.356)$ & 0.321 & $0.565(0.267-1.198)$ & 0.137 \\
\hline \multicolumn{5}{|l|}{ AJCC TNM stage } \\
\hline I & Reference & & Reference & \\
\hline II & $1.573(0.938-2.640)$ & 0.086 & $1.304(0.636-2.673)$ & 0.468 \\
\hline III-IV & $3.209(2.079-4.954)$ & $<0.001$ & $3.838(2.138-6.891)$ & $<0.001$ \\
\hline
\end{tabular}

CKAP4 mRNA low- and high-level groups were stratified by the median value of CKAP4 mRNA expression. CKAP4, cytoskeleton-associated membrane protein 4; OS, overall survival; DFS: Disease-free survival; HR, hazard ratio; AJCC, American Joint Committee on Cancer; NA, not applicable, sample size too small $(\mathrm{n} \leq 5)$ to perform univariate Cox regression analysis; TNM, tumor node metastasis. 
was significantly elevated in patients with HCC compared with healthy controls, and with patients with chronic hepatitis B and cirrhosis; the study also revealed that $86 \%$ of AFP-negative patients with HCC displayed elevated serum CKAP4 levels. Notably, the previously mentioned study reported that a combined biomarker panel of AFP and CKAP4 presents improved diagnostic accuracy for HCC compared with stand-alone analysis of either AFP or CKAP4, even in diagnosing early HCC. Additionally, a good association has been observed between CKAP4 mRNA and protein expression in tissues from patients with HCC (19). These findings indicate that both CKAP4 mRNA in tumor tissue and CKAP4 protein in serum are potential biomarkers for $\mathrm{HCC}$ detection that complement AFP evaluation.

The findings of the present study also confirmed the prognostic value of CKAP4 mRNA in patients with HCC. Univariate Cox regression analysis revealed that a high CKAP4 mRNA level was one of the three risk factors for OS and DFS in patients with HCC. In the present study, patients with HCC who had a high CKAP4 mRNA level were observed to have unfavorable OS and DFS rates (based on Kaplan-Meier survival curve analyses) and a shorter survival time or shorter recurrence time after curative surgical resection. These results are consistent with previous findings in numerous tumors (14-16) but differ from a report by Li et al (19), which demonstrated that patients with HCC and high CKAP4 mRNA (tissue microarray analysis) and protein (western blot analysis) expression had favorable OS and DFS rates. The present study considers that CKAP4 is associated with poor prognosis in HCC patients, as this conclusion is supported directly by the results of survival analysis, but also indirectly by the significant associations found between CKAP4 mRNA levels and typical prognosis-related clinicopathological factors (Table I), including histological differentiation grade, TNM stage and AFP level.

CKAP4 promotes tumor growth in numerous human cancers via different mechanisms. Kimura et al (14) demonstrated that CKAP4 expressed at the plasma membrane stimulated pancreatic and lung cancer cell proliferation by activating the phosphoinositide 3-kinase-AKT pathway; upregulation of CKAP4 was also strongly correlated with tumor growth and poor prognosis in patients. Shinno et al (16) reported that CKAP4 was upregulated in $\sim 40 \%$ of patients with ESCC and as a DKK1 receptor in cells activated the AKT pathway and promoted ESCC cell proliferation when DKK1 was highly expressed. DKK1 has also been reported to be upregulated in HCC tissues and associated with advanced tumor stage, metastasis, recurrence, OS and DFS rates $(28,29)$. However, a previous study revealed that CKAP4 inhibited HCC growth and metastasis by suppressing EGFR signaling, which indicates that the effect of CKAP4 in HCC is the opposite of that observed in the other aforementioned cancer types (20). This is also contradictory to previous findings that CKAP4 mRNA is upregulated in tissues from patients with $\mathrm{HCC}$, and significantly associated with tumor size, intrahepatic metastasis and portal venous invasion (19). More rigorous experiments are required to elucidate the effect and mechanism of CKAP4 in HCC growth and progression.

The present study has several limitations. Firstly, the HCC clinical data from the GDC TCGA-LIHC database were incomplete; thus, the sample size was insufficient for multivariate Cox regression analysis, and to subsequently conclude that CKAP4 mRNA is an independent risk factor for HCC prognosis. Secondly, although the upregulation of CKAP4 mRNA was confirmed in each of the four independent datasets, only one available dataset included clinical data that could be used to evaluate the diagnostic and prognostic significance of CKAP4 mRNA in HCC. Thus, cohorts with more patients with $\mathrm{HCC}$ are required to validate the results of the present study. Also, there were no data demonstrating CKAP4 protein expression in tumor and corresponding adjacent tissues for simultaneous analysis, although a strong correlation between CKAP4 mRNA and protein expression has been observed previously (19). Future large-scale studies without the aforementioned limitations are required to verify the findings of the present study.

In view of the controversial role of CKAP4 in HCC growth and progression, the present study extracted data from the GEO and TCGA public databases to systematically investigate the relationship between CKAP4 mRNA and HCC. The findings of the present study confirmed that significantly higher CKAP4 mRNA levels are present in tumor tissues compared with adjacent liver tissues in patients with $\mathrm{HCC}$, and that this upregulation is closely associated with poor clinicopathological conditions and outcome; this indicated the potential value of CKAP4 in HCC diagnosis and prognosis. To the best of our knowledge, this is the first study to investigate the association between CKAP4 mRNA expression in tumor tissues and the clinical characteristics of HCC based on data in public databases.

\section{Acknowledgements}

Not applicable.

\section{Funding}

No funding was received.

\section{Availability of data and materials}

The datasets analyzed during the current study are available from the corresponding author on reasonable request.

\section{Authors' contributions}

ZYC and KHZ designed the present study. TW, XG, SHC, YTH and YQW conducted the study and analyzed the data. ZYC wrote the manuscript and $\mathrm{KHZ}$ revised the manuscript. All the authors read and approved the final version of the manuscript.

\section{Ethics approval and consent to participate}

Not applicable.

\section{Patient consent for publication}

Not applicable.

\section{Competing interests}

The authors declare that they have no competing interests. 


\section{References}

1. Bray F, Ferlay J, Soerjomataram I, Siegel RL, Torre LA and Jemal A: Global cancer statistics 2018: GLOBOCAN estimates of incidence and mortality worldwide for 36 cancers in 185 countries. CA Cancer J Clin 68: 394-424, 2018.

2. Global Burden of Disease Liver Cancer Collaboration; Akinyemiju T, Abera S, Ahmed M, Alam N, Alemayohu MA, Allen C, Al-Raddadi R, Alvis-Guzman N, Amoako Y, et al: The burden of primary liver cancer and underlying etiologies from 1990 to 2015 at the global, regional, and national level: Results from the global burden of disease study 2015. JAMA Oncol 3 : 1683-1691, 2017.

3. Shimada K, Sano T, Sakamoto Y and Kosuge T: A long-term follow-up and management study of hepatocellular carcinoma patients surviving for 10 years or longer after curative hepatectomy. Cancer 104: 1939-1947, 2005.

4. Poon RT: Prevention of recurrence after resection of hepatocellular carcinoma: A daunting challenge. Hepatology 54: 757-759, 2011.

5. Tralhao JG, Dagher I, Lino T, Roudie J and Franco D: Treatment of tumour recurrence after resection of hepatocellular carcinoma. Analysis of 97 consecutive patients. Eur J Surg Oncol 33 746-751, 2007.

6. Aravalli RN, Steer CJ and Cressman EN: Molecular mechanisms of hepatocellular carcinoma. Hepatology 48: 2047-2063, 2008.

7. Gupta N, Manevich Y, Kazi AS, Tao JQ, Fisher AB and Bates SR Identification and characterization of p63 (CKAP4/ERGIC-63/ CLIMP-63), a surfactant protein A binding protein, on type II pneumocytes. Am J Physiol Lung Cell Mol Physiol 291: L436-L446, 2006.

8. Schweizer A, Ericsson M, Bachi T, Griffiths G and Hauri HP. Characterization of a novel $63 \mathrm{kDa}$ membrane protein. Implications for the organization of the ER-to-Golgi pathway. J Cell Sci 104: 671-683, 1993.

9. Nikonov AV, Hauri HP, Lauring B and Kreibich G: Climp-63-mediated binding of microtubules to the ER affects the lateral mobility of translocon complexes. J Cell Sci 120 2248-2258, 2007.

10. Shibata Y, Shemesh T, Prinz WA, Palazzo AF, Kozlov MM and Rapoport TA: Mechanisms determining the morphology of the peripheral ER. Cell 143: 774-788, 2010.

11. Bates SR, Kazi AS, Tao JQ, Yu KJ, Gonder DS, Feinstein SI and Fisher AB: Role of P63 (CKAP4) in binding of surfactant protein-A to type II pneumocytes. Am J Physiol Lung Cell Mol Physiol 295: L658-L669, 2008.

12. Conrads TP, Tocci GM, Hood BL, Zhang CO, Guo L, Koch KR, Michejda CJ, Veenstra TD and Keay SK: CKAP4/p63 is a receptor for the frizzled-8 protein-related antiproliferative factor from interstitial cystitis patients. J Biol Chem 281: 37836-37843, 2006.

13. Shahjee HM, Koch KR, Guo L, Zhang CO and Keay SK: Antiproliferative factor decreases Akt phosphorylation and alters gene expression via CKAP4 in T24 bladder carcinoma cells. J Exp Clin Cancer Res 29: 160, 2010.

14. Kimura H,Fumoto K, Shojima K, Nojima S, Osugi Y, Tomihara H, Eguchi H, Shintani Y, Endo H, Inoue M, et al: CKAP4 is a Dickkopf1 receptor and is involved in tumor progression. J Clin Invest 126: 2689-2705, 2016

15. Kikuchi A, Fumoto K and Kimura H: The Dickkopf1cytoskeleton-associated protein 4 axis creates a novel signalling pathway and may represent a molecular target for cancer therapy. Br J Pharmacol 174: 4651-4665, 2017.

16. Shinno N, Kimura H, Sada R, Takiguchi S, Mori M, Fumoto K, Doki Y and Kikuchi A: Activation of the Dickkopf1-CKAP4 pathway is associated with poor prognosis of esophageal cancer and anti-CKAP4 antibody may be a new therapeutic drug. Oncogene 37: 3471-3484, 2018.
17. Johnston HE, Carter MJ, Larrayoz M, Clarke J, Garbis SD, Oscier D, Strefford JC, Steele AJ, Walewska R and Cragg MS: Proteomics profiling of CLL versus healthy B-cells identifies putative therapeutic targets and a subtype-independent signature of spliceosome dysregulation. Mol Cell Proteomics 17: 776-791, 2018

18. Kimura H, Yamamoto H, Harada T, Fumoto K, Osugi Y, Sada R, Maehara N, Hikita H, Mori S, Eguchi H, et al: CKAP4, a DKK1 receptor, is a biomarker in exosomes derived from pancreatic cancer and a molecular target for therapy. Clin Cancer Res 25: 1936-1947, 2019.

19. Li SX, Tang GS, Zhou DX, Pan YF, Tan YX, Zhang J, Zhang B, Ding ZW, Liu LJ, Jiang TY, et al: Prognostic significance of cytoskeleton-associated membrane protein 4 and its palmitoyl acyltransferase DHHC2 in hepatocellular carcinoma. Cancer 120: 1520-1531, 2014

20. Li SX, Liu LJ, Dong LW, Shi HG, Pan YF, Tan YX, Zhang J, Zhang B, Ding ZW, Jiang TY, et al: CKAP4 inhibited growth and metastasis of hepatocellular carcinoma through regulating EGFR signaling. Tumour Biol 35: 7999-8005, 2014.

21. Wang Y, Yu W, He M, Huang Y, Wang M and Zhu J: Serum cytoskeleton-associated protein 4 as a biomarker for the diagnosis of hepatocellular carcinoma. Onco Targets Ther 12: 359-364, 2018.

22. Cao Y, Ding W, Zhang J, Gao Q, Yang H, Cao W, Wang Z, Fang L and Du R: Significant down-regulation of urea cycle generates clinically relevant proteomic signature in hepatocellular carcinoma patients with macrovascular invasion. J Proteome Res 18: 2032-2044, 2019.

23. Ramalho FS, Ramalho LN, Della Porta L and Zucoloto S: Comparative immunohistochemical expression of p63 in human cholangiocarcinoma and hepatocellular carcinoma. J Gastroenterol Hepatol 21: 1276-1280, 2006.

24. Grinchuk OV, Yenamandra SP, Iyer R, Singh M, Lee HK, Lim KH, Chow PK and Kuznetsov VA: Tumor-adjacent tissue co-expression profile analysis reveals pro-oncogenic ribosomal gene signature for prognosis of resectable hepatocellular carcinoma. Mol Oncol 12: 89-113, 2018.

25. Wang H, Huo X, Yang XR, He J, Cheng L, Wang N, Deng X, Jin H, Wang N, Wang C, et al: STAT3-mediated upregulation of lncRNA HOXD-AS1 as a ceRNA facilitates liver cancer metastasis by regulating SOX4. Mol Cancer 16: 136, 2017.

26. Mah WC, Thurnherr T, Chow PK, Chung AY, Ooi LL, Toh HC, The BT, Saunthararajah Y and Lee CG: Methylation profiles reveal distinct subgroup of hepatocellular carcinoma patients with poor prognosis. PLoS One 9: e104158, 2014.

27. Li MH, Dong LW, Li SX, Tang GS, Pan YF, Zhang J, Wang H, Zhou HB, Tan YX, Hu HP and Wang HY: Expression of cytoskeleton-associated protein 4 is related to lymphatic metastasis and indicates prognosis of intrahepatic cholangiocarcinoma patients after surgery resection. Cancer Lett 337: 248-253, 2013.

28. Desert R, Mebarki S, Desille M, Sicard M, Lavergne E, Renaud S Bergeat D, Sulpice L, Perret C, Turlin B, et al: 'Fibrous nests' in human hepatocellular carcinoma express a Wnt-induced gene signature associated with poor clinical outcome. Int J Biochem Cell Biol 81: 195-207, 2016.

29. Huang Y, Yang X, Zhao F, Shen Q, Wang Z, Lv X, Hu B, Yu B, Fan J and Qin W: Overexpression of Dickkopf-1 predicts poor prognosis for patients with hepatocellular carcinoma after orthotopic liver transplantation by promoting cancer metastasis and recurrence. Med Oncol 31: 966, 2014.

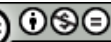

This work is licensed under a Creative Commons Attribution-NonCommercial-NoDerivatives 4.0 International (CC BY-NC-ND 4.0) License. 